\title{
EFFECT OF STRESS ON HAND MOVEMENT IN A LABORATORY SETTING AMONG HIGH SCHOOL STUDENTS: PRELIMINARY RESEARCH
}

original paper

(c) Wroclaw University of Health and Sport Sciences

DOI: https://doi.org/10.5114/hm.2021.103870

\section{HYUNGSOOK KIM ${ }^{1,2,3}$, DAVID O’SULLIVAN ${ }^{4}$, ANTONIO CAMURRI ${ }^{5}$, YONGHYUN PARK ${ }^{3}$, KSENIA KOLYKHALOVA ${ }^{5}$, STEFANO PIANA ${ }^{5}$, JEONG AE YOU ${ }^{6}$, HEE SEONG JEONG ${ }^{7}$}

${ }^{1}$ Department of Cognitive Sciences, School of Intelligence, Hanyang University, Seoul, Republic of Korea

${ }^{2}$ Graduate School of Public Policy, Hanyang University, Seoul, Republic of Korea

${ }^{3}$ HY Digital Healthcare Center, Hanyang University, Seoul, Republic of Korea

${ }^{4}$ Department of Sports Science, Pusan National University, Pusan, Republic of Korea

${ }^{5}$ Casa Paganini InfoMus Research Centre, Department of Informatics, Bioengineering, Robotics, and Systems Engineering,

University of Genoa, Genoa, Italy

${ }^{6}$ Department of Physical Education, Chung-Ang University, Seoul, Republic of Korea

${ }^{7}$ Department of Sports and Health Management, Mokwon University, Daejeon, Republic of Korea

\begin{abstract}
Purpose. The purpose of this study was to investigate the effect of stress on movement before and after a computer application-based simulated stress task.

Methods. Differences in the movement of participants were examined by measuring movement quality described by wrist accumulated distance, velocity, acceleration, jerk, and smoothness. Ten high school students performed 3 horizontal and vertical hand circling movements before and after a simulated concentration-based stress task. Blood pressure was measured, and a saliva sample was collected before and after the stress test execution. The participants were instructed to take a 10-minute relaxation period, perform 3 horizontal and vertical circling movements, a 20-minute stress task, and then perform 3 horizontal and vertical movements.

Results. There were significant differences between before- and after-stress-task levels of cortisol $(p<0.05)$, heart rate $(p<0.01)$, smoothness $(p<0.01)$, and jerk movements $(p<0.05)$ in the vertical plane. The stress-related variables were lower after the relaxation phase than the stress task. Likewise, movements were smoother and had less jerk in the vertical plane after relaxation.
\end{abstract}

Conclusions. This study indicates that stress may affect hand movement quality in the vertical plane. Therefore, we recommend that any movement behaviour adaptive therapy should focus on movements in the vertical plane.

Key words: movement, health behaviour, stress, Laban analysis, behaviour

\section{Introduction}

Korea is known for its high technology and rapid development; however, this came with a price of growing social issues, such as mental health problems (depression, anxiety, learning disabilities, and aggressive behaviour). Furthermore, as a result of the fast economic growth, the Korean society has become ultra-competitive in many social arenas, especially the academic sector, which has a high suicidal rate [1]. According to the Organization for Economic Co-operation and Development [2], Korea produces high-ranking students in mathematics and science. However, its educational system is reported to be one of the most significant stressors of depression and suicide in youths (7.8 per 100,000 people) [1]. Academic stress at high school is elevated owing to the difficulties of entrance into a highranking university, making career choices, student's

Correspondence address: David Michael O’Sullivan, Department of Sports Science, Pusan National University, 2 , Busandaehak-ro 63beon-gil, Geumjeong-gu, Pusan, 46241, Rep. of Korea, e-mail: davidosullivan@pusan.ac.kr

Received: May 22, 2020

Accepted for publication: December 8, 2020

Citation: Kim H, O’Sullivan D, Camurri A, Park Y, Kolykhalova K, Piana S, You JA, Jeong HS. Effect of stress on hand movement in a laboratory setting among high school students: preliminary research. Hum Mov. 2022;23(2):28-37; doi: https://doi.org/10.5114/hm.2021.103870. 
low academic achievement, amount of academic work, and a lack of rest [1]. The pressure to perform successfully at school is a heavy burden for adolescents, as there is a strong Korean culture of having high expectations and an aspiration to accomplish high academic scores [3]. More importantly, this time of high stress and consequently poor academic performance is reported to be a major trigger for depression, anxiety, and suicidal ideation [3, 4].

With the high importance that students place on academic achievement to get into a good university, engagement in class and high levels of concentration are reported to be strong predictors of academic achievement [5]. The authors conclude that for engagement to occur, concentration is a critical factor, and if stress exists, it can have a negative effect on concentration levels. Similarly, Lee [6] suggests that educational interventions should focus on improving student concentration, whereas other research states that maintaining a stress-free environment is vital for effective academic performance [7].

In this study, we were interested in how stress could affect movement and how movement could affect stress. Our hypothesis was that movement could affect stress, as in the recent findings explained by Shafir [8] that movement could regulate emotion, i.e. motor behaviour, such as engaging specific facial expressions, posture, and whole-body movements. We developed a horizontal and vertical plane hand circling movement as we were interested in how stress affected movement in both the horizontal and vertical planes. Upward movements have been associated with emotions such as joy, surprise, and admiration [9]. Moreover, there is an increase in emerging research describing the ability of motion capture systems to quantify emotions by analysing certain movement qualities [9]. These automatic emotion classifying systems are based on the movement qualities and posture positions, such as an open and closed posture, which are associated with different emotional states [9]. The movement using the upper body segments was selected because breathing is known to affect stress levels and therefore would influence the movement of the scapula and arms [10].

Lefter et al. [11] have developed and investigated novel methods of measuring stress from human-human interactions by analysing audio-visual recordings. Moreover, automatic methods were developed to recognize stress from semantic messages, such as spoken words for speech and meaningful gestures, and stress conveyance by the modulation of speech intonation and the speed and rhythm of gestures. A distinct advantage of the methods developed by Lefter et al. [11] for measuring stress is that they are non-invasive and can be applied in numerous situations. Thus, in this study, we hypothesized that stress could affect movement characteristics (movement distance and kinematics) and movement quality (smoothness). With this hypothesis, our ultimate aim is to understand how stress during concentration tasks alters movement characteristics. By knowing how movement might be affected by stress, we plan to develop and test the bidirectional relationship between movement behavioural correction methods and their ability to reduce stress in additional studies.

\section{Material and methods}

\section{Participants}

The group of participants, high school students, included a total number of 10 people (mean age: $15.7 \pm$ 0.48 years, mean height: $1.7 \pm 0.08 \mathrm{~m}$, mean weight: $68.3 \pm 13.2 \mathrm{~kg}$ ) recruited from a local high school. The recruitment procedure was public and a poster was placed on the high school notice board after permission was obtained from the school.

\section{Stress task development}

Common stress tasks, such as the Trier Social Stress Test and the Sing-a-Song Stress Test, were deemed unsuitable for high school students; as many stress tasks are commonly criticized for their lack of potency as a stressor [12], we developed our own one. Upon the advice of a group of 5 local psychology experts (content validity ratio $>0.99$ for $n=5$ ) [13], we developed our stress task (total: 20 minutes) by mixing and editing the contents of 3 cognitive tasks (that were deemed essential by all experts questioned): the Korean FAIR concentration test (10 minutes, based on Gnambs and Freund [14], reliability ranging 0.9-0.94 [15]); the Korean Stroop Test (3 minutes, reliability Cronbach's alpha: 0.78 [16]); and filling in a $10 \times 10$ concentration grid task (7 minutes, 1-week test-retest reliability: 0.79 [17]). These 3 tests were selected to stimulate factors that are needed in an academic setting, i.e. concentration, attention, and selective attention. The total time for the stress task was 20 minutes as Hellhammer and Schubert [18] have reported that it takes 10-20 minutes for the saliva cortisol level to reach a maximum. Furthermore, we altered these tests and converted them from the standard paper format to a format that could be used on an iPad [19] (details are shown in Figure 1). 


\section{HUMAN MOVEMENT}

H. Kim et al., Effect of stress on movement

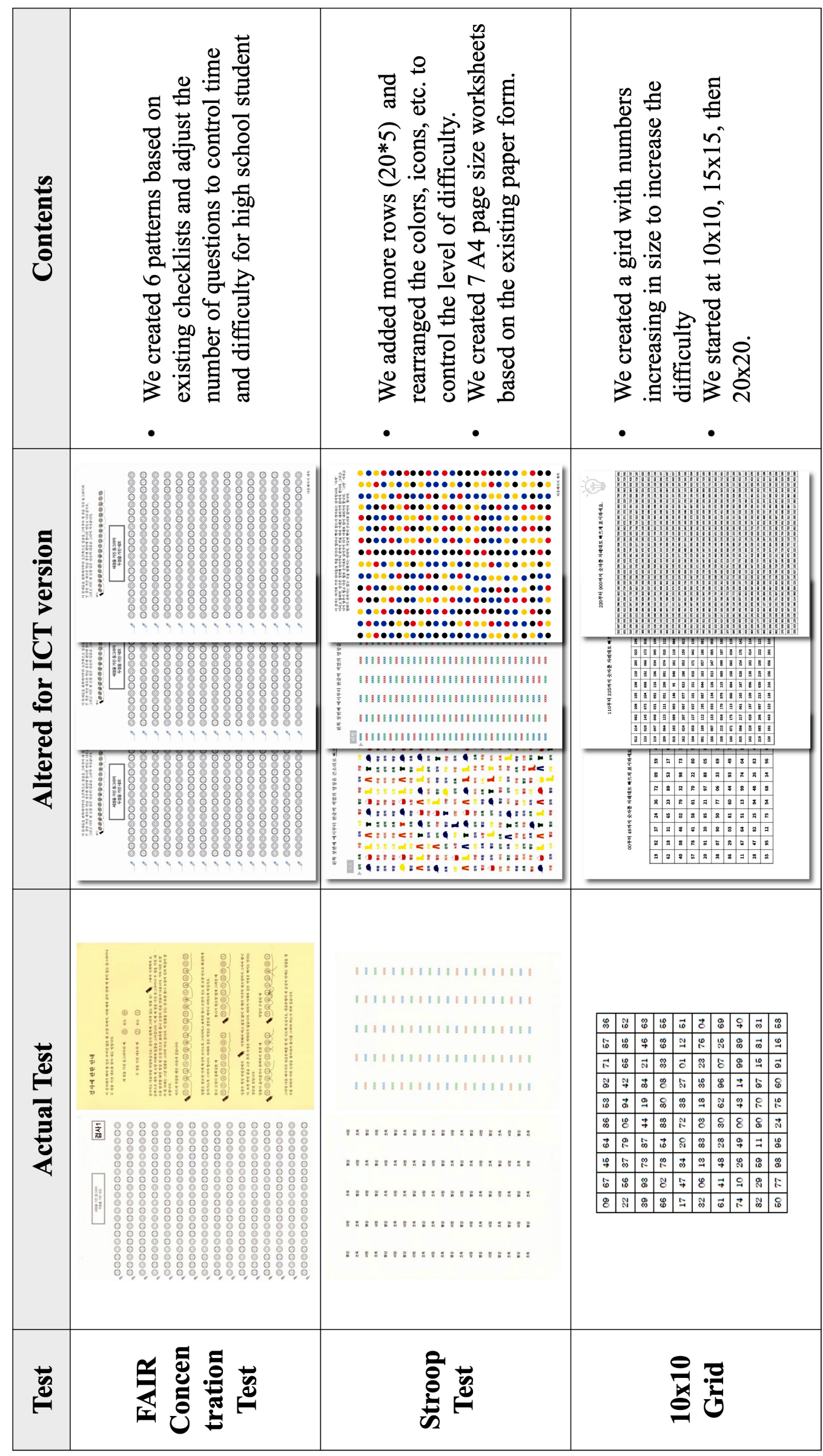

हี 
Testing procedure

The experimental setup and procedure had the following steps:

1. Participants' consent and exclusion criteria. After an introduction to the test procedure and obtaining the participants', parents', or guardians' consent, the study proceeded. To ensure that all participants had no high stakes examinations close to the time of testing, the recruitment and testing took place early in the academic school calendar. Furthermore, prior to arrival, all participants were instructed not to have any caffeinated or acidic drinks within up to 24 hours before participating in the study.

2. General stress questionnaire. The participants filled in a general stress questionnaire [20], which consisted of 21 questions and focused on their expectations, internal feelings, and expression of the stress response. The internal consistency, Cronbach's $\alpha$, of the academic stress scale ranged 0.83-0.89 [20]. The stress questionnaire was used to ensure the homogeneity of the participants. One of the 11 participants' data had to be removed because the motion capture data were corrupt.

3. Preparation and physiological data collection phase. Before the stress task was performed, the participants had their blood pressure measured (MDF Instruments, USA) and provided a sample of saliva (5 ml) (JMBio Care, Republic of Korea). Then, each subject changed into an outfit required for better data acquisition (tight-fitting top, spandex shorts) and was equipped with a heart rate monitor (Polar, Electro Oy, Kempele, Finland) chest strap.
4. Relaxing phase. The goal of the relaxation phase was to create a condition in which the participants would be able to relax. The students were instructed to go to a designated area (enclosed for privacy) to relax sitting with their eyes closed on a large bean-bag; during this relaxing 10-minute period, the participants' heart rate was continuously monitored [21].

5. Motion capture data acquisition and stress task phase. The participants were fitted with 11 reflective markers $(25 \mathrm{~mm})$ on the upper body (left and right shoulder, elbow, wrist, forehead, back of the head, and the sternum). Each student was instructed to perform a T-pose static position followed by 2 types of dynamic arm circling movements (Figure 2); first, in the horizontal (transverse) plane, and then in the vertical (frontal) plane. Each movement was repeated 3 times. For the horizontal movement, the left and right hand began in the anatomical position (standing straight with the palms of the hands facing anteriorly), moving in the anterior direction in the coronal plane, then performing a lateral movement (Figure 2), and returning to the anatomical position. For the vertical movement, the left and right hand began in the anatomical position, moving in the superior direction in the frontal plane, then performing a lateral movement (Figure 2), and returning to the anatomical position. The students were instructed to perform these 2 circling movements as large, smooth, and relaxed as possible, with no audio stimulation for the timing. After that, the subjects performed a stress task, sitting down at the desk. The stress task consisted of 10 minutes of the FAIR concentration test, 3 minutes of the Stroop Test, and 7 minutes of filling in a $10 \times 10$ grid. To make a more stress-
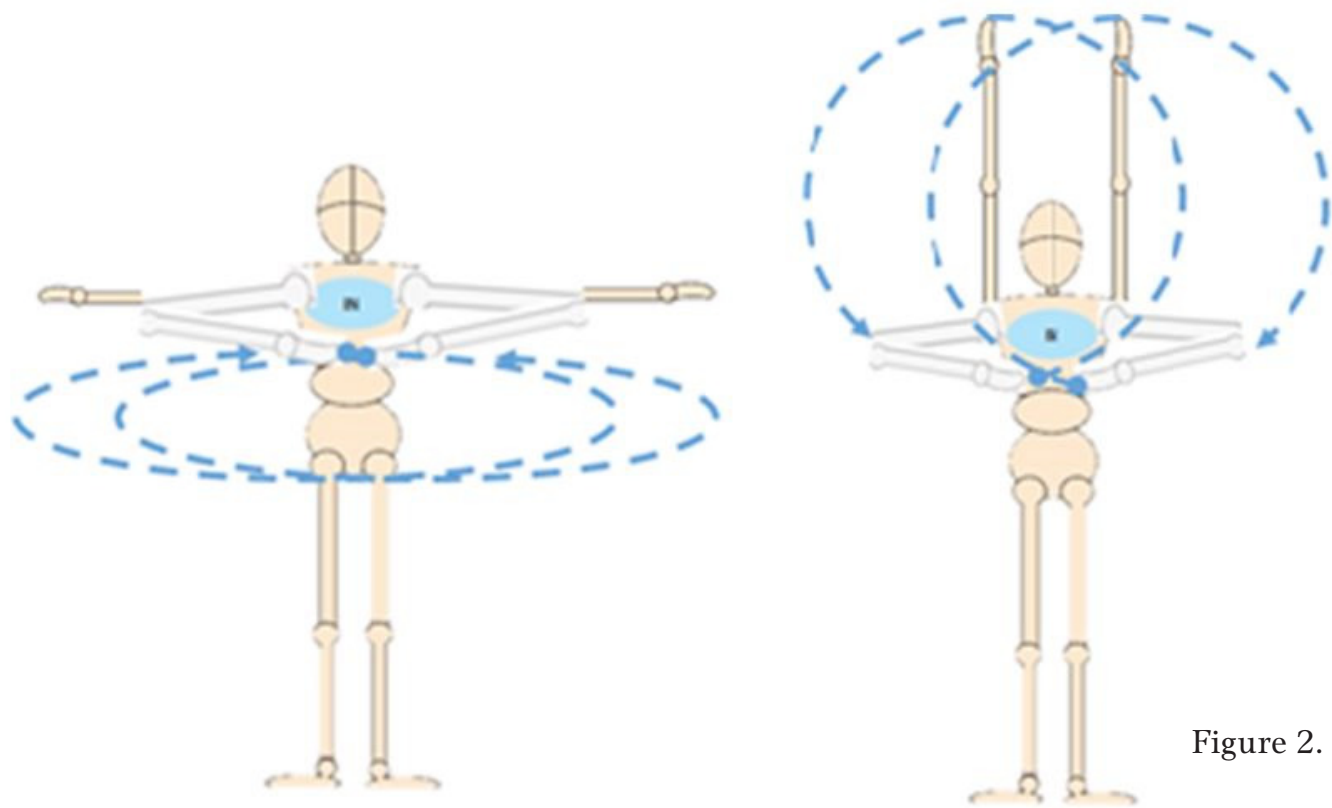

Figure 2. Horizontal and vertical arm circling motion 
ful environment, each student was instructed that their parents or guardians were watching and that they were to do their best. After the stress task, the participants were asked to perform the same circling arm movements in the horizontal (transverse) plane and in the vertical (frontal) plane as before the stress task. Directly after that, the students were requested to provide a saliva sample and had their blood pressure measured.

\section{Equipment and data processing}

Kinematic data were recorded with a 3D tracking system (OptiTrack, USA) which consisted of 12 highresolution cameras (Prime 41, USA). The positional data of reflective markers were recorded at $60 \mathrm{~Hz}$ by the OptiTrack software Motive (version 1.10.3). The data were post-processed: labelled and noise and jitter trajectories were eliminated. Afterward, the data were exported to the C3D file format for further analysis in Visual3D (C-Motion, USA). Kinematic data were filtered with a $2^{\text {nd }}$ order zero-lag low-pass filter (cutoff frequency of $8 \mathrm{~Hz}$ ), which was determined by applying a fast Fourier transform. The data are available from the corresponding author upon request.

\section{Movement features and physiological measures extraction}

In order to perform the movement analysis and explore how stress affected movement, we calculated and extracted movement features from the position of right and left wrist markers changing through time: accumulated distance, smoothness, and jerkiness. The accumulated distance was calculated as a cumulative sum of the absolute values of the displacements, the total distance travelled by the marker of the left and right wrist. Smoothness was defined as the ratio between velocity and acceleration of the normalized and averaged motion capture data. 'Smoothness value' is the value in the range $[0,1]$ (the higher the value, the higher the smoothness). Jerkiness was calculated as the rate of change of acceleration, that is, the derivative of acceleration over time, and as such it is the second derivative of velocity, or the third derivative of position. The jerk is the rate of change of acceleration and its units are meters per second cubed. The movement features were computed by using a Motion Capture Toolbox in MATLAB for computational analysis of movement data.

For physiological data, heart rate (bpm), systolic blood pressure $(\mathrm{mm} \mathrm{Hg})$, diastolic blood pressure (mm Hg), and cortisol concentration $(\mu \mathrm{g} / \mathrm{dl})$ were recorded. This study detailed various physiological factors to strengthen the validity of the stress level measurement owing to the differences in stress depending on personality types. Heart rate data were recorded with a Polar sensor (RS400, Electro Oy, Kempele, Finland) at a frequency of $100 \mathrm{~Hz}$ during the entire testing period. Blood pressure was measured manually by the same research assistant, who was specifically trained for internal consistency. Salvia samples were taken by the same research assistant and stored in an icebox, which was delivered on the next day to an independent company (JMBio Care, Republic of Korea) for analysis.

\section{Statistical methods}

The statistical analysis was carried out by using the SPSS software (version 23.0). The before- and aftertask data were calculated; then, a paired $t$-test was used to investigate if there were statistical differences, with a significance level of $p<0.05$. To further investigate the meaning of the significant differences, we followed with the recommendations by Dunlap et al. [22] for calculating the effect size of the paired $t$-test to see how large the significant differences were. On the basis of Cohen's effect size recommendations, the $d$ value of 0.2-0.5 stood for small effect size, $d$ of $0.5-0.8$ for medium effect size, and $d>0.8$ for large effect size [23]. Owing to the difficulty of recruiting, we had 10 participants and so to check the statistical power, we performed a post-hoc power analysis using the $G^{*}$ Power software, with the sample size of 10 and the alpha level of 0.05 , which resulted in a power of 0.839 , deemed an acceptable power according to Cohen [23].

\section{Ethical approval}

The research related to human use has complied with all the relevant national regulations and institutional policies, has followed the tenets of the Declaration of Helsinki, and has been approved by the $\mathrm{Pu}$ san National University Institutional Review Board (PNU\# 2017_33_HR).

\section{Informed consent}

Informed consent has been obtained from all individuals included in this study and from their legal guardians. 


\section{Results}

The paired $t$-test showed significant differences between the status before and after the stress task for the systolic and diastolic blood pressure. Among the physiological variables, there were significant differences with medium to strong effect sizes between the levels of cortisol $(t=2.35, p<0.04, d=0.61)$ and heart rate $(t=3.93, p<0.003, d=0.53)$ before and after the stress task. However, there were no significant differences in blood pressure (Table 1).

Among the kinematic variables from the motion data, smoothness and jerkiness showed statistical differences only for the vertical hand circling movement. Among the kinematic data, there were significant differences with medium to strong effect sizes for smoothness (left wrist: $p<0.008, d=0.67$; right wrist: $p<$ $0.042, d=0.41$ ) and jerk (left wrist: $p<0.023, d=0.82$; right wrist: not significant) during the vertical hand circling movement. No significant differences were reported for any of the variables, such as accumulated distance, velocity, acceleration, smoothness, or jerk for the horizontal hand circling movement between the status before and after the stress task (Table 2).

Table 1. Comparison of physiological data between the status before and after the stress task

\begin{tabular}{lrrcc}
\hline Parameter & \multicolumn{1}{c}{ Before } & After & \multicolumn{1}{c}{$p$} & Effect size $(d)$ \\
\hline Blood pressure - systolic $(\mathrm{mm} \mathrm{Hg})$ & $117.21 \pm 7.60$ & $116.62 \pm 10.70$ & 0.71 & NA \\
Blood pressure - diastolic $(\mathrm{mm} \mathrm{Hg})$ & $73.20 \pm 6.12$ & $72.40 \pm 9.27$ & 0.62 & NA \\
Cortisol $(\mu \mathrm{g} / \mathrm{l})$ & $3.30 \pm 0.81$ & $4.30 \pm 1.61$ & $<0.04^{*}$ & 0.61 \\
Heart rate $(\mathrm{bpm})$ & $76.50 \pm 8.87$ & $81.20 \pm 9.40$ & $<0.003^{* *}$ & 0.53 \\
\hline
\end{tabular}

Values are expressed as mean \pm standard deviation.

$\mathrm{NA}$ - not applicable

${ }^{*} p<0.05,{ }^{* *} p<0.01$

Table 2. Comparison of kinematic and movement quality data between the status before and after the stress task

\begin{tabular}{lcccc}
\hline \multirow{2}{*}{ Parameter } & \multicolumn{2}{c}{ Horizontal circling } & \multicolumn{2}{c}{ Vertical circling } \\
\cline { 2 - 5 } & Left wrist & Right wrist & Left wrist & Right wrist \\
\hline Accumulated distance $(\mathrm{m})$ & & & & \\
Before & $4.79 \pm 1.57$ & $4.65 \pm 1.82$ & $4.03 \pm 1.88$ & $4.99 \pm 1.51$ \\
After & $4.33 \pm 1.61$ & $4.11 \pm 1.38$ & $5.32 \pm 1.73$ & $4.47 \pm 1.34$ \\
$p$ & 0.609 & 0.502 & 0.160 & 0.571 \\
\hline Velocity $(\mathrm{m} / \mathrm{s})$ & & & \\
Before & $0.0361 \pm 0.04$ & $0.0291 \pm 0.03$ & $0.006 \pm 0.01$ & $0.010 \pm 0.01$ \\
After & $0.0349 \pm 0.04$ & $0.0339 \pm 0.03$ & $0.020 \pm 0.02$ & $0.0171 \pm 0.02$ \\
$p$ & 0.947 & 0.782 & 0.108 & 0.065 \\
\hline Acceleration $\left(\mathrm{m} / \mathrm{s}^{2}\right)$ & & & \\
Before & $0.0523 \pm 0.05$ & $0.0343 \pm 0.04$ & $0.0287 \pm 0.03$ & $0.0359 \pm 0.04$ \\
After & $0.0833 \pm 0.15$ & $0.0793 \pm 0.14$ & $0.0568 \pm 0.09$ & $0.0543 \pm 0.08$ \\
$p$ & 0.562 & 0.373 & 0.333 & 0.230 \\
\hline Smoothness & & & \\
Before & $0.22 \pm 0.025$ & $0.23 \pm 0.021$ & $0.27 \pm 0.045$ & $0.27 \pm 0.049$ \\
After & $0.22 \pm 0.028$ & $0.23 \pm 0.030$ & $0.24 \pm 0.032$ & $0.25 \pm 0.027$ \\
$p$ & 0.939 & 0.871 & $0.008^{* *}$ & $0.042^{*}$ \\
Effect size & & & 0.67 & 0.41 \\
\hline Jerk $\left(\mathrm{m} / \mathrm{s}^{3}\right)$ & & & \\
Before & $30.71 \pm 8.66$ & $27.33 \pm 7.99$ & $24.53 \pm 8.89$ & $25.07 \pm 9.26$ \\
After & $34.61 \pm 12.36$ & $30.75 \pm 8.34$ & $31.87 \pm 9.61$ & $29.09 \pm 9.91$ \\
$p$ & 0.245 & 0.167 & $0.023^{*}$ & 0.161 \\
Effect size & & & 0.82 & \\
\hline Vars & & &
\end{tabular}

Values are expressed as mean \pm standard deviation.

$\mathrm{NA}$ - not applicable

${ }^{*} p<0.05,{ }^{* *} p<0.01$ 


\section{Discussion}

In summary, this study demonstrates how a simulated stress task may influence the movement quality among high school students in a laboratory setting. The kinematic results in this study show that a stress task can affect the smoothness and the jerk of the movement. Interestingly, before the stress task was employed, the movements could be described as more efficient, as the movement distance tended to be reduced, i.e. less mechanical work was performed; however, this was not statistically significant. Similar to skill levels in sports and dance, more skilled athletes and dancers' movements are reported to be smoother and have less jerk (golf [24], dancing [25]). Likewise, the participants' upward hand circling movements were smoother after the relaxation period at the start, and had lower jerk values than after the stress task. Furthermore, high jerk values in the movement have been linked with various health issues, such as Parkinson's disease [26] and poor gait function [27].

Among the physiological data, cortisol level and heart rate indicate that the participants' stress level increased as a result of our stress task as both parameters increased significantly (moderate to strong effect size) [28]. There was not a significant effect of the stress task on the systolic or diastolic blood pressure. There seem to be some contradictions in the published research as some of the stress-related studies show an increase in the blood pressure [29], whereas some present no significant effects [30]. In an interesting study, the authors demonstrated no significant differences between the blood pressure and heart rate between stress and control conditions. Remarkably, just performing one 30-minute session of hatha yoga was reported to significantly accelerate the recovery of blood pressure and heart rate after doing a paced auditory serial addition test as a psychological stress task [31]. Moreover, as stress is known to affect breathing patterns and potentially induce different emotional states [32], we hypothesized that stress could be reduced by controlling breathing, which might decrease muscle tension and thus help the movement to become more smooth.

Another study, which measured heart rate variability and blood pressure during a mental stress task in the course of computer work [33], showed that there were significant differences between the heart rate variability during rest and stress task. Contradictory to our study, Hjortskov et al. [33] observed differences in blood pressure during different testing periods as a response to combined physical and mental workloads, as well as during and after periods of rest. Our study did not reveal any significant variations in the blood pressure but there was an overall lowering of systolic blood pressure after the stress task, which can be viewed positively in an educational setting as it indicates that the participants were more relaxed as soon as the stress task was finished, and is linked to better memory function [34]. A study investigating the effect that conflict had on cortisol levels reported a rapid reduction of cortisol level after the conflict was terminated [35], which might explain the lack of significant blood pressure differences between the status before and after the stress task. However, at this moment, we are unsure why the stress task affected the cortisol levels and heart rate, but not the systolic or diastolic blood pressure. Further studies should be carried out to investigate the relationship between different stressors and individual responses across varied age groups, i.e. from teenagers to older adults.

It is important to mention the various limitations of this study. Creating a stress task that is not too severe and therefore allows to obtain an institutional review board approval is difficult [12]. Previous research has employed several tests, which have a very narrow application, such as the Trier Social Stress Test [36] or Sing-a-Song Stress Test [37]. In the implementation of these tests, research highlights the large variation in the psychological and physiological effects of individual participants' responses as a limitation of their usage [36]. In this study, we were focused on investigating the effect of academic type stress situations which require concentration/attention during the stress task, so these existing tests (Trier Social Stress Test and Sing-a-Song Stress Test) would not have been suitable. The present study can be perceived as a preliminary investigation of the use of analysing movement quality characteristics to detect stress early; by understanding the dimensions in which movement is effected, a stress relief procedure may be developed engaging movement in the affected dimensions. The data in this study show that the stress task affected the movement pattern in the vertical plane but did not affect it in the horizontal plane; therefore, we could develop a movement-based intervention focusing on movement in the vertical (frontal) plane to help reduce stress. In psychological research on removing a nervous habit and tics [38], the majority of nervous movements tended to be upward movements of the limbs, i.e. shoulder jerking, as well as nail-biting, head jerking, and eyelash plucking. To remove these habits and tics, the authors 
recommended and tested their theory of behavioural pattern opposite corrective exercises, such as depressing the shoulders, tensing of the neck, holding the hands and shoulders down. Interestingly, these pattern opposite exercises were reported to be effective in 11 of the 12 participants of the study. We believe that the correlations between the physiological states and movement characteristics and movement quality data may be helpful to establish more detailed automated behavioural analysis techniques for an infield high school classroom-based study.

A major limitation was the difficulty of recruiting more than 10 students to participate in the study. Even though the Institutional Review Board consent was received from the University Hospital, it was very problematic to acquire the 3 parties involved (the school principal, the students, and their parents/guardians) to agree to partake in the research. As the majority of high school students in Korea attend after-school institutes for additional study and classes up until 10:00 or 11:00 p.m. during school days, all the testing had to be carried out during the weekend, which made the students even more resistant to participate. We had planned to test 30 individuals but even after recruiting students for 3 months, we were only able to recruit 10 subjects.

As stress affects both physiological and movement characteristics and movement quality, advanced computer vision techniques may be developed to analyse these so that they can be applied in stressful environments, such as schools, hospitals, flight control centres, where the tracking of the patrons' stress level would be useful for improving quality of care and provide continuous monitoring of the psychological status. Therefore, non-invasive behaviour-based stress analysis can be a very useful method of detecting people's stressbased behaviours early. On the basis of the relationship between stress and behaviour, Lefter et al. [11] are developing a system that can recognize stress through the analysis of semantics and modulation of speech and associated gestures. They highlight the difficulty of measuring stress through behaviour as it is conveyed by a large variety of combinations of various communicative acts. Furthermore, the authors suggest that it is easy to misinterpret a behaviour without considering the overall gestures and other nonverbal cues such as postures, facial expressions, and body language.

\section{Conclusions}

This study shows that our stress task affected the stress level and movement quality in male and female high school students. Secondly, we indicate that stress reactions to a simulated stress task can be measured by physiological methods, i.e. changes in heart rate and cortisol levels. More importantly, the data reveal that stress affects our movement quality, which can be measured by movement features calculated from motion capture data, such as smoothness and jerkiness of the movements. This study illustrates the possibility to implement passive motion capture systems as a diagnostic tool for evaluating mental (stress) and physical health through the measurement of movement quality. In future studies, we plan to apply these movement quality analysis techniques in a high school classroom during class to see if there is a difference in movement quality depending on students' stress levels.

\section{Acknowledgements}

This work was supported by the Global Research Network program through the Ministry of Education of the Republic of Korea and the National Research Foundation of Korea (NRF-2016S1A2A2912583).

\section{Disclosure statement}

No author has any financial interest or received any financial benefit from this research.

\section{Conflict of interest}

The authors state no conflict of interest.

\section{References}

1. Lee S-Y, Hong JS, Espelage DL. An ecological understanding of youth suicide in South Korea. Sch Psychol Int. 2010;31(5):531-546; doi: 10.1177/014303431038 2724.

2. OECD. Education at a glance 2014: Korea. Available from: https://www.oecd.org/education/Korea-EAG2014 -Country-Note.pdf.

3. Park S-M, Cho S-I, Moon S-S. Factors associated with suicidal ideation: role of emotional and instrumental support. J Psychosom Res. 2010;69(4):389-397; doi: 10.1016/j.jpsychores.2010.03.002.

4. Park HS, Schepp KG, Jang EH, Koo HY. Predictors of suicidal ideation among high school students by gender in South Korea. J Sch Health. 2006;76(5):181-188; doi: 10.1111/j.1746-1561.2006.00092.x.

5. Casuso-Holgado MJ, Cuesta-Vargas AI, Moreno-Morales N, Labajos-Manzanares MT, Barón-López FJ, Vega-Cuesta M. The association between academic engagement and achievement in health sciences students. BMC Med Educ. 2013;13(1):33; doi: 10.1186/ 1472-6920-13-33.

6. Lee JS. The relationship between student engagement and academic performance: is it a myth or reality? 
J Educ Res. 2014;107(3):177-185; doi: 10.1080/00220 671.2013.807491.

7. Fredricks JA, Blumenfeld PC, Paris AH. School engagement: potential of the concept, state of the evidence. Rev Educ Res. 2004;74(1):59-109; doi: 10.3102/0034 6543074001059 .

8. Shafir T. Using movement to regulate emotion: neurophysiological findings and their application in psychotherapy. Front Psychol. 2016;7:1451; doi: 10.3389/ fpsyg.2016.01451.

9. Piana S, Staglianò A, Camurri A, Odone F. A set of fullbody movement features for emotion recognition to help children affected by autism spectrum condition. IDGEI International Workshop. 2013.

10. Schleifer LM, Ley R, Spalding TW. A hyperventilation theory of job stress and musculoskeletal disorders. Am J Ind Med. 2002;41(5):420-432; doi: 10.1002/ajim. 10061.

11. Lefter I, Burghouts GJ, Rothkrantz LJM. Recognizing stress using semantics and modulation of speech and gestures. IEEE Trans Affect Comput. 2016;7(2):162175; doi: 10.1109/TAFFC.2015.2451622.

12. Jayasinghe SU, Torres SJ, Nowson CA, Tilbrook AJ, Turner AI. Physiological responses to psychological stress: importance of adiposity in men aged 50-70 years. Endocr Connect. 2014;3(3):110-119; doi: 10.1530/ EC-14-0042.

13. Lawshe CH. A quantitative approach to content validity. PersPsychol.1975;28(4):563-575; doi: 10.1111/j.17446570.1975.tb01393.x.

14. Gnambs T, Freund M. NEPS technical report for attention: administration of the Frankfurt Attention Inventory (FAIR) in starting cohort 4 (grade 9) for students with special educational needs. Bamberg: Leibniz Institute for Educational Trajectories; 2019.

15. Ahn J-D, Han N-I, Kim J-W. Concentration variation through sport talented children's training program [in Korean]. J Korea Contents Assoc. 2012;12(8):343354; doi: 10.5392/JKCA.2012.12.08.343.

16. Kim TY, Kim SY, Sohn JE, Lee EA, Yoo BG, Lee SC, et al. Development of the Korean Stroop Test and study of the validity and the reliability [in Korean]. J Korean Geriatr Soc. 2004;8(4):233-240.

17. Heinen T. Do static-sport athletes and dynamic-sport athletes differ in their visual focused attention? Sport J. 2011;14(1).

18. Hellhammer J, Schubert M. The physiological response to Trier Social Stress Test relates to subjective measures of stress during but not before or after the test. Psychoneuroendocrinology. 2012;37(1):119-124; doi: 10.1016/j.psyneuen.2011.05.012.

19. Bajaj JS, Heuman DM, Sterling RK, Sanyal AJ, Siddiqui M, Matherly S, et al. Validation of EncephalApp, smartphone-based Stroop Test, for the diagnosis of covert hepatic encephalopathy. Clin Gastroenterol Hepatol. 2015;13(10):1828-1835.e1; doi: 10.1016/j.cgh. 2014.05.011.
20. Lee J, Puig A, Kim Y-B, Shin H, Lee JH, Lee SM. Academic burnout profiles in Korean adolescents. Stress Health. 2010;26(5):404-416; doi: 10.1002/smi.1312.

21. Prinsloo GE, Derman WE, Lambert MI, Rauch HGL. The effect of a single session of short duration biofeedback-induced deep breathing on measures of heart rate variability during laboratory-induced cognitive stress: a pilot study. Appl Psychophysiol Biofeedback. 2013; 38(2):81-90; doi: 10.1007/s10484-013-9210-0.

22. Dunlap WP, Cortina JM, Vaslow JB, Burke MJ. Metaanalysis of experiments with matched groups or repeated measures designs. Psychol Methods. 1996;1(2): 170-177; doi: 10.1037/1082-989X.1.2.170.

23. Cohen J. Statistical power analysis for the behavioral sciences. New York: Lawrence Erlbaum Associates; 1988.

24. Choi A, Joo S-B, Oh E, Mun JH. Kinematic evaluation of movement smoothness in golf: relationship between the normalized jerk cost of body joints and the clubhead. Biomed Eng Online. 2014;13(1):20; doi: 10.1186/1475925X-13-20.

25. Camurri A, Canepa C, Ferrari N, Mancini M, Niewiadomski R, Piana S, et al. A system to support the learning of movement qualities in dance: a case study on dynamic symmetry. Proceedings of the 2016 ACM International Joint Conference on Pervasive and Ubiquitous Computing: Adjunct. 2016;973-976; doi: 10.1145/ 2968219.2968261.

26. Baron EI, Koop MM, Streicher MC, Rosenfeldt AB, Alberts JL. Altered kinematics of arm swing in Parkinson's disease patients indicates declines in gait under dual-task conditions. Parkinsonism Relat Disord. 2018; 48:61-67; doi: 10.1016/j.parkreldis.2017.12.017.

27. Tack GR, Choi JS, Yi JH, Kim CH. Relationship between jerk cost function and energy consumption during walking. In: Magjarevic R, Nagel JH (eds.), World Congress on Medical Physics and Biomedical Engineering 2006. IFMBE Proceedings, vol. 14. Berlin, Heidelberg: Springer; 2007; 2917-2918.

28. Hillier A, Murphy D, Ferrara C. A pilot study: shortterm reduction in salivary cortisol following low level physical exercise and relaxation among adolescents and young adults on the autism spectrum. Stress Health. 2011;27(5):395-402; doi: 10.1002/smi.1391.

29. Conley KM, Lehman BJ. Test anxiety and cardiovascular responses to daily academic stressors. Stress Health. 2012;28(1):41-50; doi: 10.1002/smi.1399.

30. Kim W-J, Kwon M-H, Kwon M-H, Kim J-G. Effects of aroma therapy on EEG and academic stress [in Korean]. Sci Emot Sensib. 2015;18(1):95-102; doi: 10.14695/ KJSOS.2015.18.1.95.

31. Benvenutti MJ, da Sliva Alves E, Michael S, Ding D, Stamatakis E, Edwards KM. A single session of hatha yoga improves stress reactivity and recovery after an acute psychological stress task - a counterbalanced, randomized-crossover trial in healthy individuals. Complement Ther Med. 2017;35:120-126; doi: 10.1016/j. ctim.2017.10.009. 
32. Philippot P, Chapelle G, Blairy S. Respiratory feedback in the generation of emotion. Cogn Emot. 2002;16(5): 605-627; doi: 10.1080/02699930143000392.

33. Hjortskov N, Rissén D, Blangsted AK, Fallentin N, Lundberg U, Søgaard K. The effect of mental stress on heart rate variability and blood pressure during computer work. Eur J Appl Physiol. 2004;92(1-2):84-89; doi: 10.1007/s00421-004-1055-z.

34. Galvin JA, Benson H, Deckro GR, Fricchione GL, Dusek JA. The relaxation response: reducing stress and improving cognition in healthy aging adults. Complement Ther Clin Pract. 2006;12(3):186-191; doi: 10.1016/j. ctcp.2006.02.004.

35. Powers SI, Pietromonaco PR, Gunlicks M, Sayer A. Dating couples' attachment styles and patterns of cortisol reactivity and recovery in response to a relationship conflict. J Pers Soc Psychol. 2006;90(4):613-628; doi: 10.1037/0022-3514.90.4.613.

36. Williams RA, Hagerty BM, Brooks G. Trier Social Stress Test: a method for use in nursing research. Nurs Res. 2004;53(4):277-280; doi: 10.1097/00006199200407000-00011.

37. Brouwer A-M, Hogervorst MA. A new paradigm to induce mental stress: the Sing-a-Song Stress Test (SSST). Front Neurosci. 2014;8:224; doi: 10.3389/fnins.2014. 00224.

38. Azrin NH, Nunn RG. Habit-reversal: a method of eliminating nervous habits and tics. Behav Res Ther. 1973; 11(4):619-628; doi: 10.1016/0005-7967(73)90119-8. 